\title{
A defence of the welfarist account of enhancement
}

\author{
Hazem Zohny
}

Bioethics Centre, Division of Health Sciences, University of Otago, PO Box 56, Dunedin, 9054, New Zealand Hazem.zohny@gmail.com 
*Highlights

\section{Highlights}

- The welfarist account of enhancement and its implications are presented

- Objections against this account are argued to be either unfounded or addressable

- This account offers a coherent, normative approach to thinking about enhancement 


\section{A defence of the welfarist account of enhancement}

What do we mean by the term "enhancement"? Imagine taking a performance-enhancing drug that allows you to solve math problems a lot faster than you used to. If your math skills were previously average, we could say that the drug has enhanced you in that respect. If your math skills were previously very poor (say, due to some identifiable cognitive dysfunction), and now you are able to solve maths problems at an average speed, we could say the drug has treated you. Based on this distinction, an enhancement seems to be a biomedical intervention that goes beyond the ends of medicine in what is necessary to sustain or restore good health or normal functioning. It's a common definition of enhancement [1-4].

Alternatively, we can ignore whether your math skills are average or poor and just focus on the fact that the drug boosts your skills compared to how they were before. In that sense, enhancement can be understood in terms of its augmentative impact on your capacities, regardless of whether they are below or above a certain standard of functioning relative to a given population [5-7].

There are other possible ways of defining enhancement (see $[8,9])$. However, I am interested in a particular account that understands the term as a change in the body or mind that tends to improve one's well-being. This is the welfarist account of enhancement, largely developed by Julian Savulescu and colleagues [9-11]. It defines an enhancement as:

"Any change in the biology or psychology of a person which increases the chances of leading a good life in the relevant set of circumstances." [9]

Based on this definition, the performance-enhancing drug envisioned above is not necessarily enhancing at all. This is because, whether it qualifies as enhancing or not depends on whether having better math skills would likely contribute to your well-being - that is, to your chances of leading a good life in your set of circumstances, which I understand to refer to one's particular societal and personal context.

Here, I want to focus on some of the criticisms levelled against this definition of enhancement. To be clear, by including "any change in the biology or psychology of a person" in its definition, the welfarist account disregards the distinction between what we might think of as science or technology-based interventions in the body (such as taking a drug or inserting a brain implant), and more conventional interventions (such as exercise or education). "Any change" also entails that whether the intervention is for treating a dysfunction or for augmenting an already well-functioning capacity, both can be considered enhancements - so long as they tend to increase well-being. As such, therapeutic treatments are rendered a subclass of more general enhancing interventions.

Because of this, the welfarist account has been criticized on multiple grounds. Sparrow [12] argues that these implications mean that the term becomes very broad, losing contact with the everyday language use of enhancement. Coenen and colleagues similarly suggest that enhancement may become a useless term if it fails to highlight science-based interventions and distinguish them from more conventional ones [13]. They also argue that having a concept of enhancement that allows us to clearly distinguish it from therapeutic interventions will be key to effective policy-making.

Other criticisms have centred on its reliance on the contested notion of "a good life" and well-being more generally. Beck and Stroop argue that this account will not be helpful in classifying interventions as enhancing or not due to the variety of conceptions of well-being [14]. There is also a worry that, by defining enhancement as whatever will likely contribute to well-being, this can act like a philosophical sleight of hand that appears to settle the ethics of enhancement: if to enhance is to 
increase well-being, then it may seem difficult to conceive of anything objectionable about enhancement $[12,14]$. Further criticisms claim that this account risks underestimating the social and cultural pressures that influence individual choices, as well as fails to take into account the social and collective consequences of enhancing $[8,15]$.

These criticisms are considerable. However, I argue here that they are either unfounded or addressable. First, it's worth noting the temptation of thinking that we don't need to have an explicit and shared definition of what enhancement is in order to have a meaningful conversation about it. The lack of such a consensus certainly hasn't stopped the burgeoning enhancement literature. But note that how we understand concepts can have major consequences for how we view them ethically, and therefore how we regulate them. For instance, whether we understand a foetus as a soul-endowed person or a lump of cells will have major implications for what we think we can do with them (abort them, source them for stem cells, etc.). Similarly, whether we understand enhancements as interventions that are mere excesses that go beyond restoring normal functioning, or as interventions that contribute to well-being, will have significant implications on how we regulate their use. Because of that, we need a coherent, shared understanding of the enhancement. Becoming clearer about what the welfarist account entails exactly is a step in that direction.

\section{The welfarist account of enhancement}

A helpful way of understanding the welfarist account is to start by making a distinction between functional enhancement and human enhancement [9]. Enhancement in the functional sense denotes some improvement in a capacity. It says nothing about the effect of that improvement on the person undergoing it. In general, we tend to associate improved capacities with positive effects on our quality of life. Better memory, more resilient immune systems, stronger bodies - these can implicitly suggest a corresponding improvement in the quality of one's life.

But we can also see how that won't always be the case. If having improved memory led to a greater propensity to developing phobias, or if improved hearing led to being constantly distracted, these may well result in a diminishment to one's qualify of life. Similarly, we can see how diminishing our capacities to recall certain traumatic events, or even our ability to hear if we lived in an incredibly noisy environment - these might lead to an increase in one's quality of life, even though they would be the opposite of functional enhancement [16]. More generally, none of the alterations to these cognitive or sensory systems would be relevant to quality of life if the person undergoing them is in a permanently vegetative state.

It is this discrepancy between functional enhancement and quality of life that prompts the notion of human enhancement. Here, it is not the change to our capacities that constitutes the enhancement, it's the positive impact of that change on our well-being, which I take to be synonymous with leading a good life. Hence, if super hearing abilities were to lead a person to constant distraction, thereby reducing their well-being, that would not constitute an instance of human enhancement. Similarly, if someone suffering from PTSD diminished their ability to recollect certain memories and thereby increased their well-being, that would be an instance of human enhancement.

This is why the welfarist definition of human enhancement emphasises any change in the biology or psychology or a person: it does not have to be a strictly functional improvement.

This also relates to the account's emphasis on the "relevant set of circumstances" of the person undergoing an intervention - which is to say, the person's particular context. What this entails is that there is no context-independent answer to the question of whether an intervention is an enhancement. The circumstances of the individual undergoing an intervention play a determining 
role in whether that intervention will likely be enhancing. In the case of the drug that boosts maths skills envisioned above, if one works as an accountant, it may be enhancing for them, even if it had the side-effect of, say, reducing their musical abilities. On the other hand, if a musician who rarely works with numbers used the drug, it may be difficult to see how the drug is enhancing, given their particular circumstances.

Note that these elaborations highlight how this is an account that defines what an enhancement is it is not an account of whether that enhancement should be made use of. That is, the fact that a biological or psychological change may be enhancing in the sense of increasing well-being, is not to say that it should be undergone. Depending on the alternatives, it may be that changing the environment instead of the individuals' biology or psychology will be more reasonable or practical. Moreover, it may be that making use of an enhancement will result in harm to others or it may create or exacerbate injustice more generally. The welfarist account is compatible with these possibilities: the fact that a modification is enhancing does not necessarily mean that it should be made use of.

As we will now see, many of the objections to the welfarist account noted above lose their bite once we have properly understood the implications of this approach.

\section{Renders "enhancement" too broad}

This criticism notes that, by focusing on any change to a person's biology or psychology, the welfarist definition doesn't distinguish between science or technology-based enhancements into the body such as drugs or genetic manipulations - and more conventional enhancements - such as exercise or education [17]. Coenen and colleagues argue that there are qualitative differences between the means used in science-based and conventional enhancements, and that these should not be defined away [13]. While they don't clarify what those qualitative differences are precisely, Wagner and colleagues elaborate on them by contrasting cognitive enhancement drugs with education [18].

They acknowledge that, like drugs, education impacts synaptic connections and neural networks in the brain. However, they argue that education does so in a way that is more stable and long lasting. This is because, unlike the effect of drugs, neural changes due to education are the result of repetition and practice over an extended time frame. They argue that a definition of enhancement that ignores such differences risks committing a fallacy of ambiguity [18].

While Wagner and colleagues are focusing specifically on pharmacological cognitive enhancement, their argument relates to a broader intuition that science-based enhancements like drugs work in a way that is transient and effortless, while conventional enhancements like education and training require effort and have more stable and long lasting effects. However, this dichotomy between transient and effortless versus enduring and effortful does not hold up.

Let's first consider the case of transience. One the one hand, it's certainly conceivable that future cognitive enhancing drugs will have longer lasting or potentially permanent effects. For instance, a single high dose of the hallucinogen psilocybin (the active ingredient in magic mushrooms) can create lasting personality changes [19]. Beyond drugs, the effects of genetic modifications would be anything but transitory. Similarly, bodily or neural prosthetics entail a degree of permanence. In contrast, despite the effort and repetition involved in studying, few go on to remember the details of a class mere weeks after exams. And despite the effort involved in endurance training, fitness levels start to plummet in as little as two weeks of not training [20]. Which is to say, conventionally gained knowledge and fitness can be transient too. 
What about the claim that science-based enhancements entail less effort? Again, we can consider a number of counter-examples. Firstly, taking a putative cognitive enhancing drug will not, in and of itself, lead to performing better in, say, an exam. Study is still required: we still have to learn the relevant information. Similarly, taking steroids has to be combined with training to build muscles, and individuals fitted with prosthetics or a brain-machine interface need weeks of practice before they can use them properly. In contrast, it's not always clear that conventional enhancement involves much effort: watching entertaining documentaries can be highly educational, yet it barely requires effort (unless we are talking about a more superficial kind of effort, such as dedicating time to watching, in which case we can also talk about the effort of remembering to take a pill every day or the effort of hooking up a brain-machine interface).

More generally, it is not clear what makes an intervention science or technology-based to begin with. Modern education and training have become deeply intertwined with technology, and so has how we produce our food - good nutrition having obvious enhancing effects on our bodies and minds.

Nevertheless, it is true that things like transience and effort can be morally relevant in this context. Investing in a life that is built around transient effects along with a diminished sense of accomplishment may be a less valuable sort of life. ${ }^{1}$ But while we may be disposed to thinking that science-based interventions raise more pressing questions about health safety, authenticity, accomplishment, societal values, distributive justice, and the host of other ethical issues associated with enhancement technologies, these questions are also relevant to so-called conventional enhancements. For instance, we can ask questions about the health safety of intensive study, the stress and sedentary lifestyle it entails being issues of growing health concern. Also, education can change people in profound, irreversible and often rapid ways, and hence it may also raises questions related to authenticity and societal values. In fact, reading a single book can have profound and irreversible effects on a person. More broadly, how we regulate education, access to nutrition, and healthcare - all things that can have life-changing enhancing effects on individuals - has significant implications for fairness and distributive justice.

And so, the fact that these issues - safety, authenticity, societal values, and justice - may appear to arise in seemingly novel or pressing ways when thinking about current or hypothetical interventions associated with science-based enhancements doesn't make them qualitatively different issues. The sentiment that they are is a misplaced one, and the welfarist definition helps us sidestep that false intuition. In that sense, the account keeps the focus on what is actually relevant: the impact of interventions on our lives rather that the means behind those interventions.

\section{Fails to distinguish therapy from enhancement}

A similar criticism focuses on the welfarist definition's lack of a distinction between therapy and enhancement. Coenen and colleagues describe the importance of that distinction as the key conceptual challenge in policy-making contexts, allowing us, for instance, to prioritize the relevant sorts of interventions in terms of what we research and fund [13]. Given limited resources, most would be inclined to argue that therapy should be prioritized over enhancement since being diseased or injured is more likely to stop an individual from being a normal competitor able to lead a

\footnotetext{
${ }^{1}$ Although, note that if investing in a life built around transient effects and a diminished sense of accomplishment reduces the chances of leading a good life, interventions that led to such a diminishment would not be considered enhancements based on the welfarist account.
} 
good life. Prioritizing therapy is therefore central to maintaining basic standards of equal opportunity [2].

The welfarist definition of enhancement appears to eschew this challenge, demoting therapeutic treatments of disease and injury to a subclass of other interventions that tend to increase well-being in a given context. Hence, whether one is mending a broken arm, or cutting off a healthy arm and replacing it with a stronger, bionic one, both are enhancements so long as they tend to increase well-being in the relevant circumstances.

Adding to that line of thought, some have argued that if we don't make a distinction between therapy and enhancement, it may be too easy to argue that all forms of enhancement are morally permissible given that the things we count as therapy are permissible [21]. However, it's not clear that just because something counts as therapy it is deemed permissible. Its permissibility is contingent on the circumstances of the person undergoing it. For instance, it would not be permissible to administer chemotherapy to someone who doesn't have cancer. Therapeutic treatments, especially risky ones with adverse side effects, are generally only permissible when we have reason to believe that they will make the life of the person undergoing it go better. That, in the broadest terms, is our guiding criterion for such decisions.

And this is where the welfarist account is most useful. Because, while it may not provide a clear-cut distinction between therapy and enhancement, it provides a more relevant distinction: interventions to the body and/or mind that tend to contribute to well-being, and those that don't. As it happens, things that we think of as therapies tend to contribute more to our well-being than interventions that might improve our functioning beyond some norm. That is, in most circumstances, mending a broken limb or treating cancer contributes far more to our chances of leading a good life than, say, augmenting normal eye sight or concentration levels. Under the welfarist account, this gives us concrete, normative reasons to prioritize such therapies over such enhancements. However, this will not always be the case. Some therapies will have little impact on the quality of our lives (say, a treatment for mild eczema), while some enhancements may have deeply positive impacts (say, enhancing one's self-control). The welfarist account allows us to say that, in some cases, developing and funding interventions that are not technically restorative of health may be prioritized over some nominally therapeutic interventions. And that is because of their impact on well-being.

To that extent, the welfarist definition again keeps us focused on what actually matters: not on nominal and hazy distinctions between interventions, but on how those interventions impact wellbeing. However, in highlighting the centrality of well-being, this brings us to a more fundamental objection to this account.

\section{It is too dependent on the contested concept of well-being.}

This criticism is well articulated by Beck and Stroop [14]:

"This account, however, seems too widely based to provide a clear definition, for whether or not something counts as human enhancement under this description depends on how we understand the notion of a 'good life'. Alternative theories of well-being and the specific aspects on which they lay emphasis are, however, likely to result in divergent classifications."

In other words, since there is no consensus about what constitutes leading a good life, there can be no consensus about what sorts of interventions are enhancing. Savulescu and colleagues [9] acknowledge that whether an intervention can be understood as enhancing will depend on the 
account of well-being we employ. But is the existence of competing accounts of well-being as problematic as Beck and Stoop suggest?

First, it is worth highlighting that some interventions are clearly enhancing on any plausible conception of a good life. This is the case for the majority of interventions that aim to restore health or prevent disease. In practice, we will likely be able to identify a wide range of interventions as either enhancing or harmful to an individual without much controversy.

There is a reason for this: while the main theories of well-being-hedonism, desire-satisfaction, and objective list theories - offer conflicting accounts of the nature of well-being, they nevertheless converge to a large extent on the things that can make a life go better or worse. In fact, at least for hedonism and desire-satisfaction, their aim is to converge. Consider things like accomplishment, friendship, autonomy, and knowledge. Hedonism and desire-satisfaction theories typically hold these as 'good' because, for most people, they give us pleasure or satisfy our desires. Objective list theories in turn tend to recognize the value of pleasure and typically hold that informed desires often reflect what is independently good [22].

This convergence is not to deny that there will be difficult cases related to whether an intervention will be enhancing for a person. However, there is no reason to believe that these will be more difficult than cases where it is unclear whether a treatment for an illness will pay off for an individual. Health and disease are indeterminate concepts - in fact, there are no less than eight different disease concepts held by individuals engaging with clinical reasoning and practice [23] - yet that generally does not pose a significant problem for determining who to treat and for what.

However, there is a related problem to the objection raised here that has not received much attention. Even if we were to form a consensus on the right account of well-being, there is still the problem of how we measure the well-being of individuals. To be sure, this is a problem for any welfarist account, not just in regards to enhancement. The problem is this: we know that individuals are fallible. People can simply be mistaken about what is good for them [24]. Related to this problem is that, to the extent that well-being entails a subjective component based on a person's evaluation of the goodness of their life, there appears to be two ways to measure that evaluation. We can speak broadly of life satisfaction, which implies reflecting back at an experience or series of experiences related to one's life, and mood or emotional well-being, which relates to how we feel during an experience as it unfolds. These two modes of evaluating our well-being can diverge quite significantly, so much so that we can speak of individuals as having a 'remembering self' and an 'experiencing self' [25].

To illustrate this, consider the relationship between income and life satisfaction, and income and emotional well-being. While life satisfaction continues to increase as one's income increases, emotional well-being does not. According to one study in the US, unlike life satisfaction, emotional well-being stops correlating with annual income once it exceeds $\$ 75,000$ [26]. Another illustrative study compared women in Columbus, Ohio, with those in Rennes, France, and found that even though American women reported higher levels of life satisfaction, French women reported spending more time in positive moods and doing the sorts of things that both French and American women agreed yielded more pleasure [27]. Whose life should we say is going better?

This discrepancy between the evaluations of our live satisfaction compared to our day to day emotional well-being may pose a problem for the welfarist account of enhancement. It raises the question of whose well-being enhancements are meant to be improving: the remembering self or the experiencing self? It is the remembering self that ultimately makes decisions. But the 
remembering self is not a very good judge of what contributes to well-being and what doesn't especially when it comes to unpleasant experiences. A study illustrating this phenomenon had participants immersing one of their hands for one minute in water cooled at $14 \mathrm{C}$ - which is considered painful. After, they immersed their other hand for 1 minute in water at $14 \mathrm{C}$, followed by a further 30 seconds during which the water was gradually warmed to $15 \mathrm{C}$. Despite the added 30 seconds of still painfully cold water, participants were more willing to repeat the second experience [28]. This is because the last 30 seconds of slightly warmer water coloured their perception of the whole experience.

This, along with similar studies $[29,30]$, reflects our bias towards giving too much weight to the last moments of an experience. In the context of human enhancement, this raises concerns not only about our ability to decide what is actually best for us, but also in what ways we are aiming to improve the quality of our lives exactly - in ways that are primarily experienced, or remembered?

Presumably, the account of well-being that we adopt should be able to answer this question, and it's likely to be one that can accommodate both of these selves and their sometimes conflicting interests. In reality, though, it's likely that most things that contribute to our emotional well-being will also contribute to our life satisfaction, and vice versa. It's also worth pointing out that our fallibility about our own well-being is not as troubling for this account as it might seem. It may be tempting to think that such fallibility opens the door for paternalism or coercion about enhancement (or any other issue, for that matter). However, by rejecting the idea that any person is infallible about well-being, we thereby also reject the idea that a would-be paternalist is infallible on the matter as well [31]. In that sense, acknowledging our fallibilism can make paternalism on these matters harder to justify.

More importantly, a welfarist account that is sensitive to our fallibility is one that, to an extent, is less likely to miss important elements of our well-being. Acknowledging our fallibility and becoming aware of the conflicting ways in which we can be wrong about what is good for us should be a part of the process of deliberating about which interventions tend to contribute to leading good lives. When a welfarist account takes these issues into consideration, it is more useful than approaches to enhancement that disregard well-being altogether. Ultimately, the problem of cognitive biases and flaws in our reasoning about what will improve our well-being is an opportunity for this account. If we develop interventions that can reduce these cognitive errors, then that would a crucial step towards increasing the chances of leading a good life in a very broad way.

\section{Prematurely settles the ethical debate}

Sparrow argues that the welfarist account risks settling the complex ethical issues of enhancement through some philosophical sleight-of-hand [12]. If enhancement is not qualitatively different from therapy or more conventional enhancements like exercise, what's all the ethical fuss about? Beck and Stroop voice this worry as:

"It is questionable whether the wide understanding of enhancement, which the welfarist account offers, properly meets the moral concerns critics of enhancement usually articulate. If everything that actually contributes to (the chances of) leading a good life is considered as enhancement, then it is not obvious how enhancement could be in the least objectionable." [14]

Similarly, Camporesi argues that the welfarist definition's focus on individual well-being neglects the broader societal and collective consequences of enhancement [8]. 
However, these concerns appear to mistakenly assume that by singling out the value of well-being at the individual level, the welfarist account does so in an exclusive way - as though other values, such as justice, are negligible. But this is false.

The welfarist approach is not meant to resolve the question of whether an enhancement is ethically permissible, it is merely meant to reframe our understanding of the concept of enhancement in a way that ties it to the well-being of the individual undergoing a modification. Well-being is a prudential good, and in that sense this account aims to capture why an enhancement is good for the person enhanced. But by singling out well-being in its definition, it does not follow that it is the only relevant thing when it comes to thinking about the permissibility of a given enhancement.

As Savulescu and colleagues state: "The welfarist approach distinguishes ways in which some treatment might benefit a person from other relevant values, such as justice. It thus allows us to say that although some treatment is an enhancement (i.e. contributes to an individual's well-being), it might nevertheless be bad overall, because its employment in the current social context will lead to far greater injustice." [9]

In that sense, considerations about other people's well-being, societal impacts, distributive justice, and whatever else a moral theory might be concerned with aside from prudential good, are still relevant considerations. Highlighting individual well-being merely keeps the definition focused away from the terminological controversies and conceptual confusions of the other accounts. It instead keeps the debate focused on what is most relevant in making sense of the relationship between an intervention and a person undergoing it: its impact on that person's well-being.

However, it is understandable where this criticism arises from. Indeed, the crux of the welfarist approach is that it transforms the question of when to enhance to when we should increase wellbeing. Met with such a question, it can be tempting to immediately answer: whenever we can.

This indeed appears to be Savulescu's response to that question: "When enhancement is understood as an intervention which increases the chances of a person having a good life, it is hard to see how there could be any objections to trying to make people's lives go better. Indeed, the fact that enhancements increase well-being provides a strong moral obligation based on beneficence to provide them." [10]

If well-being is a state of mind that is independent of any reliance on shared resources, then Savulescu may well be correct here. But well-being, however conceived, is arguably dependent to some extent on access to various external goods: at the least, shelter, healthcare, and so on. It moreover depends on what one is already used to: shelter in the form of a cave for most people living in the $21^{\text {st }}$ century would be significantly diminishing to their well-being. Given this, well-being seems to share a close relationship with limited goods. How much of those goods one appropriates has implications for how much is left for others, and in that sense, the level of well-being of an individual has implications for the well-being of others. This brings up issues of distributive justice. How should access to the sorts of goods conducive to well-being be distributed? The answer to this question depends on the account of justice employed. At this stage, things do get complicated. But at least with the welfarist account, they get complicated in a relevant way - that is, by keeping us focused on what is at stake: the well-being of individuals.

\section{Underestimates social forces}

This final objection I'll consider here relates to the criticism by Camporesi that the welfarist account appears to underestimate the social and cultural pressures that influence individual choice and how 
we define well-being [8]. For instance, in critiquing the welfarist account, Gordon argues that merely asking whether an intervention will increase well-being doesn't go far enough [15]. We also have to ask i) what social conditions have led us to consider a particular intervention as an enhancement as something that will tend to increase the chances of leading a good life? And ii) what social structures are enabled by the adoption of a given enhancement?

These are important questions, but while they are certainly relevant to an assessment of the permissibility of an enhancement, it's not clear that they are relevant to how we should define enhancement. To explain why, let us assume that the social conditions that are moulding how we define well-being are nefarious ones. For instance, we can imagine a hyper-capitalist society where the basic goods that tend to be conducive to well-being can only be attained by becoming a highly productive contributor to the economy. Here, employees operate under social forces that pressure them to become ruthless competitors who devote their entire lives to getting ahead, ceaselessly producing and consuming, with no time to enjoy family or recreation.

Indeed, such a dystopian scenario is not too far from the reality of an increasing number of industries. In such a context, we can argue that negative social pressures are redefining what the pursuit of well-being entails, and therefore, under the welfarist account, what enhancements are. Here, enhancements appear to be interventions that would improve one's ability to compete within that dubious socio-economic set up. If so, there may be two problems here.

One is that individuals within such a context might be pressured to use enhancements aimed at keeping them competitive - if they don't want to use them they will be less able to maintain their current level of well-being, let alone increase it. As for those who want to use these enhancements but can't afford them, this would be unfair to them as well as potentially unjust. However, why is this a problem for the welfarist account? As we've seen, the fact that this account highlights wellbeing does not mean that it does so at the expense of other values. If a society is structured in a way so that increasing one's well-being must come at an unjust cost of diminishing another's, then we may be required to restrict access to enhancements. The welfarist account is perfectly compatible with such a conclusion.

The other problem might be that, under such a scenario, social forces have redefined well-being into something that appears to be quite impoverished: the good life here entails becoming a ruthless competitor that spends their life contributing to an ethically suspect socio-economic setup. However, the reason we find this vision of well-being disturbing is because it is difficult to imagine how it can be called a vision of well-being to begin with. ${ }^{2}$ Something has clearly gone amiss. Members of that society are no longer actually working towards increasing their well-being in any plausible sense - rather, they appear to be stuck doing something that actually diminishes it. Or, at least, they are stuck doing something that may significantly diminish their well-being if they stopped. In that regard, this society has simply made an acceptable level of well-being very difficult, and therefore interventions that are presented as enhancements in that society are not actually enhancing in the welfarist sense. That is, they are not enhancements at all.

Hence, contrary to the concerns by Camporesi and Gordon, it's not clear how the welfarist account underestimates the relationship between social forces and one's conception of well-being. Negative social forces can lead to a context where the use of enhancements is unjust, or where the notion of well-being has become highly contaminated and therefore no longer related to any plausible

\footnotetext{
${ }^{2}$ Indeed, under such a scenario a good life might entail becoming a radical non-conformist who aims to destabilize the system - to the extent that doing so might lead to a more autonomous, meaningful, or even pleasurable life.
} 
conception of a good life. In the former case, the welfarist account is compatible with restricting the use of enhancements, and in the latter, what society deems as enhancements are not actually enhancements at all.

\section{Conclusion and further thoughts}

The welfarist account has been criticized for obscuring the distinction between science-based interventions and so-called conventional ones, as well as the distinction between therapy and enhancement. It has also been objected to on the basis that it relies on the contested concept of well-being; that it is unhelpful in classifying interventions; that it appears to settle ethical questions about enhancement through some philosophical sleight-of-hand; and that it underestimates the role social forces play in how we define well-being, as well as the collective consequences of enhancement.

I have argued that these criticisms are either unfounded or addressable. The distinction between science-based interventions and conventional ones, and the distinction between therapy and enhancement, are not actually useful, only serving to distract from what is actually of relevance: the well-being of the individual undergoing a modification. Moreover, while well-being may resist precise definition, it remains useful and open to re-evaluation, with the different theories of wellbeing converging on host of goods deemed central to a good life. The welfarist account also does not prematurely settle the ethical issues of enhancement: while it focuses debate on the nature of wellbeing, it does not do so in an exclusive way, allowing for other values to also determine whether an enhancement should be permissible. Finally, while social forces may negatively influence how we define or pursue well-being, this is not a problem for the welfarist account: such an influence will mean that either using enhancements should be restricted because they lead to injustice, or enhancements are no longer possible as society has made leading a good life impossible.

Nevertheless, more work is needed to flesh out the full implications of the welfarist account. One question is how much weight we should place on an individual's "relevant set of circumstances" in defining what an enhancement is. For instance, imagine a person who is discriminated against because their society is racist. They hear of a new intervention that can change their skin colour and allow them to avoid being discriminated against. They undergo the intervention and subsequently end up leading a better life with more prospects. In such a case, their relevant circumstances is a racist society, and the intervention they underwent appears to be enhancing. But do we want to define enhancement in a way whereby, say, a black person who becomes white can be deemed "enhanced" because of their particular circumstances? That is, should prejudicial social forces play a role in defining what might constitute an enhancement for a person? These questions point to the need for more clarity about what qualifies as the "relevant set of circumstances" exactly.

Ultimately, if the welfarist account holds up, it would involve a lot of empirical work being carried out. The number of interventions being called enhancers is growing, especially amongst the off-label use of certain drugs and brain stimulation techniques. The welfarist account entails that we now have to ask if these putative enhancers improve the well-being of their users. That is, it impels us to ask if they actually are enhancers.

\section{References}

[1] Juengst E. What does enhancement mean? In: Parens E, editor. Enhancing Human Traits: Ethical and Social Implications, Georgetown: Georgetown University Press; 1998.

[2] Daniels N. Normal Functioning and the Treatment-Enhancement Distinction. Cambridge Quarterly of Healthcare Ethics. 9 (2000) 309-22. 
[3] Resnik DB. The Moral Significance of the Therapy-Enhancement Distinction in Human Genetics. Cambridge Quarterly of Healthcare Ethics 2000;9:365-77.

[4] Pellegrino E. Biotechnology, Human Enhancement, and the Ends of Medicine 2004. https://cbhd.org/content/biotechnology-human-enhancement-and-ends-medicine (accessed May 7, 2015).

[5] Engelbart, D. Augmenting Human Intellect: A conceptual Framework. Stanford Research Inst Melo Park CA, 1962.

[6] Bostrom N, Roache R. Ethical issues in human enhancement. New Waves in Applied Ethics 2008:120-52.

[7] Harris J. Enhancing Evolution: The Ethical Case for Making Better People. Princeton, NJ: Princeton University Press; 2007.

[8] Camporesi S. From Bench to Bedside, to Track \& Field. San Francisco, CA: University of California Medical Humanities Press; 2014.

[9] Savulescu J, Sandberg A, Kahane G. Well-Being and Enhancement. In: Savulescu J, Meulen R ter, Kahane G, editors. Enhancing Human Capacities, Blackwell Publishing Ltd; 2011, p. 1-18.

[10] Savulescu J. Justice, Fairness, and Enhancement. Annals of the New York Academy of Sciences 2006.

[11] Earp B, Sandberg A, Kahane G, Savulescu J. When is diminishment a form of enhancement? Rethinking the enhancement debate in biomedical ethics. Frontiers in Systems Neuroscience. 8 (2014).

[12] Sparrow R. Queerin' the PGD Clinic. Journal of Medical Humanity. 34 (2013) 177-96.

[13] Coenen C, Schuijff M, Smits M. The Politics of Human Enhancement and the European Union. In: Savulescu J, Meulen R ter, Kahane G, editors. Enhancing Human Capacities, Blackwell Publishing Ltd; 2011, p. 521-35.

[14] Beck B, Stroop B. A Biomedical Shortcut to (Fraudulent) Happiness? An Analysis of the Notions of Well-Being and Authenticity Underlying Objections to Mood Enhancement. In: Søraker JH, Rijt J-WV der, Boer J de, Wong P-H, Brey P, editors. Well-Being in Contemporary Society, Springer International Publishing; 2015, p. 115-34.

[15] Gordon R. Enhancement and Social Possibility. The Neuroethics Blog 2014. http://www.theneuroethicsblog.com/2014/08/enhancement-and-social-possibility.html\#more (accessed August 8, 2014).

[16] Earp B, Sandberg A, Kahane G, Savulescu J. When is diminishment a form of enhancement? Rethinking the enhancement debate in biomedical ethics. Frontiers in systems neuroscience. 8 (2014)

[17] Blackford R. What is this thing called enhancement? Talking Philosophy. 2012. http://blog.talkingphilosophy.com/?p=5663 (accessed May 7, 2015).

[18] Wagner N-F, Robinson J, Wiebking C. The Ethics of Neuroenhancement: Smart Drugs, Competition and Society. International Journal of Technoethics. 6 (2015) 1-20.

[19] MacLean K, Johnson M, Griffiths R. Mystical experiences occasioned by the hallucinogen psilocybin lead to increases in the personality domain of openness. Journal of Psychopharmacology. 25 (2011) 1453-61.

[20] Coyle E, Hemmert M, Coggan A. Effects of detraining on cardiovascular responses to exercise: role of blood volume. Journal of Applied Physiology. 60 (1986) 95-9.

[21] Lin P, Allhoff F. Untangling the Debate: The Ethics of Human Enhancement. Nanoethics. 2 (2008) 251-64.

[22] Kahane G, Savulescu J. The welfarist account of disability. In: Brownlee K, Cureton A, editors. Disability and Disadvantage, Oxford University Press; 2009, p. 15-53.

[23] Freitas R. Nanomedicine, volume 1: Basic capabilities. Georgetown, TX: 1999.

[24] Levy N. Ecological Engineering: Reshaping Our Environments to Achieve Our Goals. Philosophy \& Technology. 25 (2012) 589-604.

[25] Kahneman D. Thinking, Fast and Slow. New York: Farrar, Straus and Giroux; 2011. 
[26] Kahneman D, Deaton A. High income improves evaluation of life but not emotional well-being. PNAS. 107 (2010) 6489-93.

[27] Krueger A, Kahneman D, Fischler C, Schkade D, Schwarz N, Stone A. Time Use and Subjective Well-Being in France and the U.S. Social Indicators Research. 93 (2008) 7-18.

[28] Kahneman D, Fredrickson BL, Schreiber CA, Redelmeier DA. When More Pain Is Preferred to Less: Adding a Better End. Psychological Science. 4 (1993) 401-5.

[29] Schreiber C, Kahneman D. Determinants of the remembered utility of aversive sounds. Journal of Experimental Psychology. 129 (2000) 27-42.

[30] Redelmeier D, Kahneman D. Patients' memories of painful medical treatments: real-time and retrospective evaluations of two minimally invasive procedures. Pain. 66 (1996) 3-8.

[31] Moore A. Well-being: A philosophical basis for health services. Health Care Analysis. 2 (1994) 207-16. 